\title{
„Das Staatlich Geförderte Institut für Taubstumme” in Körmöcbánya
}

\author{
Dorottya Szepessyné Judik
}

\section{Subsidised education institution for deaf-mute people in Körmöcbánya Abstract}

The inquiry about the social mobility of deaf people in Hungary is part of the history of disability, but it also can provide useful information for other disciplines, for example for the history of education, history of living, history of culture etc.

In Hungary, the first institution for deaf-mute people's education was established in 1802 , in Vác. It aimed at making deaf people „useful members” of society, which was contrary to the popular beliefs of the medieval times. In the reform era, this single institution and its achievements in the field of social mobility were insufficient to change the negative beliefs about deaf-mute people: they were mainly beggars, or were dependants of their families. The turning point was the Austro-Hungarian Compromise in 1867. By the end of 1918, 16 institutions educated deaf-mute students all across Hungary. The opportunities of social mobility for deaf people were based on this school network.

In Felvidék, 3 institutions were established: in Pozsony, in Körmöcbánya and in Jolsva. These institutions published school bulletins every year. In my paper, I examine the bulletins of the deaf education institution of Körmöcbánya to demonstrate social mobility of the former students. The bulletins contain the list of the students, their notes, information about their families (parent's age, occupation, etc.), and statistic data about the institution.

Based on the bulletins and the contemporary newspapers I illustrate the role of education in social mobility of deaf people.

Keywords: education of deaf people; Deaf education institution of Körmöcbánya; school bulletin; social mobility

Schlüsselwörter: Gehörloseninstitut in Körmöcbánya; Schulzeugnisse; soziale Mobilität; Gehörlosenbildung

Subject-Affiliation in New CEEOL: Social Sciences - Education - History of Education

DOI: 10.36007/eruedu.2020.3.083-094 


\section{Einführung}

In Ungarn wurde im Jahr 1802 in der Stadt Vác das erste Gehörloseninstitut gegründet, das sich zum Ziel gesetzt hatte, - im Gegensatz zu der seit dem Mittelalter vorherrschenden Auffassung - die Gehörlosen zu „,nützlichen Mitgliedern” der Gesellschaft zu machen. Zur Änderung der im Reformzeitalter Ungarns den Gehörlosen gegenüber auch weiterhin verbreiteten negativen Attitüde - die Mehrheit der Gehörlosen bettelt, und ihr Lebensunterhalt wird von den Familien bestritten - erwiesen sich dieses eine Bildungsinstitut und die gesellschaftliche Mobilität seiner Schüler als zu wenig. Die Wende brachte in Ungarn die prosperierende Periode des Zeitalters des Österreichisch-Ungarischen Ausgleichs. Bis Ende 1918 stieg die Zahl der Bildungsinstitute für Gehörlose auf 16, und sie deckten die Fläche des ganzen Landes ab. Die Gründung der Gehörlosenschulen determinierte die Erhöhung der gesellschaftlichen Mobilität der gehörlosen Schülerinnen und Schüler.

In Oberungarn wurden die gehörgeschädigten Schülerinnen und Schüler in drei Gehörloseninstituten erwartet: in Pozsony [Preßburg, heute Bratislava], in Körmöcbánya [Kremnitz, heute Kremnica] und in Jolsva [Jelschau, heute Jelšava]. Diese Institute stellten in jedem Schuljahr Zeugnisse aus. In der vorliegenden Studie werden die vom Gehörloseninstitut in Körmöcbánya ausgestellten Schulzeugnisse zur Abbildung der gesellschaftlichen Mobilität in den Fokus gestellt. Der Quellenwert der Schulzeugnisse wird dadurch gegeben, dass sie unter anderem die Namenslisten der Schülerinnen und Schüler, ihre schulische Entwicklung, Angaben über ihren familiären Hintergrund (z. B. die Beschäftigung der Eltern) und auf die Schule bezogene statistische Daten darlegen.

Mit Hilfe der genannten Quellen bzw. der Pressequellen jener Zeit kann nachvollzogen werden, welche Rolle die Bildung Anfang des 20. Jahrhunderts bei der gesellschaftlichen Mobilität der in Oberungarn lebenden Gehörlosen spielte.

\section{Statistischer Hintergrund}

Um zu verstehen, warum die Gehörloseninstitute eine wichtige Rolle spielten, lohnt es sich, die vom Ungarischen Königlichen Statistischen Amt durchgeführten Volkszählungen anzuschauen, bei deren Durchführung das Amt bei den Volkszählungen die „unter einer körperlichen und geistigen Behinderung leidende Bevölkerung”, darunter die "Taubstummen” mit Aufmerksamkeit verfolgte. In dieser Zeit wurden gehörlose Personen direkt als Taubstumme bezeichnet. Nach den Daten der Volkszählung im Jahr 1869 lebten in Ungarn (Kroatien-Slawonien und Fiume ausgenommen) 20699 gehörlose Personen. Hinsichtlich ihrer Lebensführung wurden zu jener Zeit noch keine Daten erhoben. (Népszámlálás 1869.) Zur Zeit der Volkszählung des Jahres 1880 lebten 19874 gehörlose Personen in Ungarn, von denen 10455 Personen ohne Beschäftigung waren, 4336 Personen arbeiteten im Ackerbau, und 2036 gaben an, im Haushalt zu arbeiten. Man muss noch hinzufügen, dass $95 \%$ der Personen, die die Angaben machten, weder lesen noch schreiben konnten. (Népszámlálás, 1880) 10 Jahre später verminderte sich das 
Verhältnis zwischen den Personen, die nicht lesen und schreiben konnten, um nur $2 \%$. Nach den Daten der Volkszählung des Jahres 1890 lebten in Ungarn 16 073 Gehörlose, davon waren 5170 Personen Kinder im Alter von 7-15 Jahren, die dazu befähigt waren, unterrichtet zu werden. (Népszámlálás, 1890) Nach den Daten der Volkszählung des Jahres 1900 lebten 22103 gehörlose Personen in Ungarn. Wenn wir uns die Zahlen der in den Komitaten Oberungarns gezählten Gehörlosen anschauen, erhalten wir folgende Daten:

\begin{tabular}{|l|c|}
\hline Name des Komitats, der Stadt & Zahl der Gehörlosen \\
\hline Ára & 378 \\
\hline Bars & 173 \\
\hline Esztergom & 155 \\
\hline Hont & 132 \\
\hline Selmecbánya* & 46 \\
\hline Liptó & 114 \\
\hline Nógrád & 240 \\
\hline Nyitra & 550 \\
\hline Pozsony & 611 \\
\hline Pozsony* & 49 \\
\hline Trencsén & 686 \\
\hline Turóc & 102 \\
\hline Zólyom & 234 \\
\hline Abaúj-Torna & 236 \\
\hline Kassa* & 33 \\
\hline Bereg & 327 \\
\hline Borsod & 282 \\
\hline Gömör und Kishont & 229 \\
\hline Sáros & 265 \\
\hline Szepes & 258 \\
\hline Ung & 375 \\
\hline Zemplén & 592 \\
\hline Insgesamt & 6087 \\
\hline
\end{tabular}

Tabelle Nr. 1: Daten der Volkszählung des Jahres 1900, mit besonderem Blick auf die Komitate Oberungarns

Wie aus der Tabelle ersichtlich ist, lag die Zahl der gehörlosen Personen aufgrund der Volkszählung des Jahres 1900 bei 6087 Personen. Eine hervorstechend hohe Zahl fanden wir in den Komitaten Trencsén, Pozsony, Zemplén, Nyitra.

\footnotetext{
* mit gesetzgeberischem Recht ausgestattete Stadt
} 


\section{Hintergrund des Bildungswesens}

Gemäß § 2 des Gesetzesartikels Nr. 38 aus dem Jahr 1868, der über den Volksschulunterricht im öffentlichen Bildungswesen erlassen wurde, „kann das Schulamt auf den amtsärztlichen Nachweis hin die körperlich oder geistig schwachen Kinder (§ 117) für kürzere oder längere Zeit von der Schulbesuchspflicht befreien." Ein halbes Jahrhundert später (!) stellte Vidor Borbély in der Fachzeitschrift der Volksschullehrer unter Bezugnahme auf das o. g. Gesetz die Frage: „Wer sagt, dass aufgrund dieses Gesetzes ein Taubstummer verpflichtet ist, die Volksschule zu besuchen, oder nicht? (Andere Gesetze berühren nicht einmal soweit die Erziehung und den Unterricht der Taubstummen.) Einerseits wäre es gut, wenn die Schulpflicht der Taubstummen in das Gesetz hineininterpretiert werden könnte, weil sie dann infolge der schulischen Ordnung und Disziplin etwas von ihrer tierischen Wildheit verlieren würden, und an innen auch etwas von menschlichen Zügen haften bliebe. $\mathrm{Na}$, und dann würde sich so versammelt die Aufmerksamkeit eher auf sie richten, $d$. h. man muss etwas mit ihnen tun, weil sie zusammen mit Menschen gesunder Empfindung unterrichtet werden können." (Borbély 1908). Diese Frage vermittelt gut, dass der Unterricht von Gehörlosen neben zahlreichen Ergebnissen auch Probleme aufwarf.

Es ist wahr, dass im historischen Ungarn das erste Gehörloseninstitut bereits im Jahr 1802 in Vác gegründet wurde. Dieses Institut befand sich - neben den kleineren, doch früh zum Erliegen gekommenen Initiativen (Pozsony und Liptószentmiklós) - bis zum Österreichisch-Ungarischen Ausgleich im Jahr 1867 auf dem Gebiet der Gehörlosenbildung in einer Monopolstellung. (Az elemi népoktatás 1915) Dies erwies sich jedoch als zu wenig. Das Zeitalter des Dualismus brachte einen spektakulären Sprung auf dem Gebiet der Bildung. Bis 1918 wurden die gehörgeschädigten Schülerinnen und Schüler bereits in 16 Gehörloseninstituten empfangen. Der Direktor des Budapester staatlichen Gehörloseninstituts, Sándor Borbély, richtete im Jahr 1900 einen Aufruf an die gesetzgebenden Behörden und übersandte innen einen Entwurf, in dem er innen Vorschläge zur Gründung von Gehörloseninstituten unterbreitete. Die Errichtung der Schulen wurde auch durch die staatliche Teilnahme gefördert. So eröffnete im Jahr 1903 auch das Gehörloseninstitut in Körmöcbánya seine Tore. (Szepessyné Judik 2009)

\section{Das Gehörloseninstitut in Körmöcbánya}

Die Gründung eines Gehörloseninstituts in Körmöcbánya kam zuerst im November 1901 zur Sprache. Das ist auch darum wichtig, weil zu jener Zeit in Ungarn im Schuljahr 1900-1901 nur 8 Institute zur Verfügung standen (in Vác, in Budapest zwei, in Kecskemét, in Temesvár, in Kaposvár, in Arad, in Kolozsvár, wo insgesamt 494 (!) Schülerinnen und Schüler ihren Bildungsweg absolvierten. Auf der Ebene Gesamtungarns genossen mehr als 4000 gehörlose Kinder keinerlei Unterricht, und so gelangten sie später in die Lage des völligen Ausgeliefertseins. (Klis 1901)

Die Generalversammlung der freien königlichen Stadt Körmöcbánya verhan- 
delte am 26. März und am 7. April 1902 die Angelegenheit der Gründung des Instituts. Um die Unterstützung der Sache zu steigern, betraute der Minister für Religions- und Bildungsangelegenheiten, Dr. Gyula Wlassics, mit seiner Verordnung Nr. 24981/1902 die Lehrkräfte des Budapester Gehörloseninstituts, in Körmöcbánya die Möglichkeiten des Unterrichts für Gehörlose darzulegen und populär zu machen sowie die erzielten Ergebnisse mit vier aus Budapest mitgebrachten gehörlosen Schülern vorzustellen. Die positive Aufnahme der Initiative zeigte sich darin, dass das Ministerium für Religions- und Bildungsangelegenheiten in seiner Verordnung Nr. 27629/1902 mit Freude zur Kenntnis nahm, dass Körmöcbánya ein Gehörloseninstitut errichten möchte. Das Ministerium erklärte sich bereit, Lehrkräfte zu entsenden, sofern das Projekt verwirklicht werden würde. Zum Jahreswechsel 1902-1903 sicherten die Generalversammlungen der Komitate Bars und Turóc ebenfalls ihre Unterstützung der Initiative zu. Am 30. Mai 1903 wurde der Schriftsatz fertiggestellt, der die Kriterien für die Errichtung des Instituts umfasste. Das Ministerium für Religions- und Bildungsangelegenheiten genehmigte die Gründung des Instituts mit seiner Verordnung Nr. 48638/1903. Die erbetene Lehrkraft stellte das Ministerium in der Person von Kálmán Hercsuth bereit, bzw. ordnete die Verbringung von gebrauchten Möbeln des Budapester Gehörloseninstituts nach Körmöcbánya an. Mit der Leitung des über eine provisorische Stelle verfügenden Instituts betraute es Kálmán Hercsuth. (Hercsuth 1904)

Das Institut formulierte seine Ziele folgendermaßen: „Das Ziel ist, dass der Taubstumme nicht auf die Fürsorge anderer angewiesen sei, sondern seinen Lebensunterhalt von seinem eigenen Verdienst bestreiten könne. Durch die Anwendung des zweckdienlichen Erziehungs- und Unterrichtssystems des Gehörloseninstituts, d. h. mit der Verwendung der tönenden Sprache und der erforderlichen realen Kenntnisse muss der Taubstumme zur Selbstständigkeit herangebildet werden, und er muss von jener Vormundschaft befreit werden, die sein Leben auch sonst in sehr enge Schranken drängt". (Hercsuth 1904)

Das Institut nahm in erster Linie Schülerinnen und Schüler aus den Komitaten Bars, Turóc, Zólyom, Liptó, Árva, Trencsén und Nyitra auf. Im Institut wurden all jene Kinder aufgenommen, die ihr 7. Lebensjahr vollendet und ihr 10. Lebensjahr nicht vollendet hatten, über ein ,amtsärztliches Zeugnis zur Bescheinigung ihrer Taubstummheit”, bzw. über ein Impfzeugnis zum Nachweis der Pockenschutzimpfung verfügten. Einen Anspruch auf Bedürftigenhilfe hatten die Schülerinnen und Schüler nur mit einer entsprechenden Vermögensbescheinigung. Der Bildungsgang dauerte 8 Jahre.

Die folgenden Lehrfächer wurden „unter Anwendung der Methode der mündlichen Rede und Ausschluss der Gebärdensprache" unterrichtet: Ausspracheübungen mit Schreiben und Lesen, Sprachlehre, beschreibender anschaulicher Unterricht, Formulieren, Lesen, Religions- und Morallehre, Rechnen, Naturkunde, Geographie, Geschichte, Verfassungskunde, Schönschrift, Zeichnen, Fingerfertigkeitsübungen und Turnen. Die Unterrichtssprache war einheitlich Ungarisch. Die beim Unterricht der einzelnen Lehrfächer anzuwendenden Materialien und Methoden wurden aufgrund der vom Minister für Religions- und Bildungsangelegenheiten herausgegebenen Verordnungen Nr. 16822 aus dem Jahr 1900 und Nr. 17550 
aus dem Jahr 1903 in Anwendung gebracht. (Mitteilungsblatt des Gehörloseninstituts von Körmöcbánya 1905-1906) Die Zahl der Zöglinge durfte in einer Klasse bei 8-15 Personen liegen.

Im ersten Unterrichtsjahr begannen 9, im zweiten Unterrichtsjahr bereits 12 Lernende den Schulunterricht auf der ersten Klassenstufe, und diese Zahl wuchs in jedem Jahrgang bis zur maximierten Anzahl von 15 Personen. Die Klasse mit 15 Personen kam zum ersten Mal im Schuljahr 1908-1909 zustande.

Den Bildungshintergrund und Werdegang der ersten neun Schülerinnen und Schüler können wir bis zum Ende ihrer schulischen Laufbahn in den fortlaufend erscheinenden Mitteilungsblättern des Instituts verfolgen.

\begin{tabular}{|l|l|l|}
\hline Name der/s Schülerin/s & Name der Ortschaft $^{2}$ & Name des Komitats \\
\hline Bezák, Anna & Chinorán & Nyitra \\
\hline Buda, Antal & Garamhidvég & Zólyom \\
\hline Csambala, Mária & Szakolca & Nyitra \\
\hline Frlicska, Margit & Nagyszocóc & Turóc \\
\hline Nagy, Róza & Böös & Pozsony \\
\hline Oszvald, Antal & Jánoshegy & Bars \\
\hline Steiner, Dezsö & Privigye & Bars \\
\hline Szakál, Béla & Nagy-Jóka & Pozsony \\
\hline Zelienka, Paula & Necpál & Túróc \\
\hline
\end{tabular}

Tabelle Nr. 2: Die Namensliste der Schülerinnen und Schüler der ersten Klasse des Gehörloseninstituts von Körmöcbánya aus den Jahren 1903-1904

(Mitteilungsblatt des Gehörloseninstituts von Körmöcbánya 1903-1904)

\begin{tabular}{|l|l|l|l|l|l|l|l|l|}
\hline $\begin{array}{l}\text { Acker- } \\
\text { bauer }\end{array}$ & $\begin{array}{l}\text { Tage- } \\
\text { löhner }\end{array}$ & Knecht & $\begin{array}{l}\text { Stein- } \\
\text { bruch- } \\
\text { arbei- } \\
\text { ter }\end{array}$ & $\begin{array}{l}\text { Hau- } \\
\text { sierer }\end{array}$ & $\begin{array}{l}\text { Einzel- } \\
\text { händ- } \\
\text { ler }\end{array}$ & $\begin{array}{l}\text { Zim- } \\
\text { mer- } \\
\text { mann }\end{array}$ & Schmied & $\begin{array}{l}\text { Insge- } \\
\text { samt }\end{array}$ \\
\hline 2 & 1 & 1 & 1 & 1 & 1 & 1 & 1 & 9 \\
\hline
\end{tabular}

Tabelle Nr. 3: Die Beschäftigung der Eltern der Zöglinge der ersten Klasse des Gehörlosen-instituts von Körmöcbánya aus den Jahren 1903-1904 (Mitteilungsblatt des Gehörloseninstituts von Körmöcbánya 1903-1904) 


\begin{tabular}{|l|l|l|}
\hline Name der/s Schülerin/s & Name der Ortschaft ${ }^{3}$ & Name des Komitats \\
\hline Bartl, József & Szucsány & Turóc \\
\hline Belik, Mária & Bars-Ceke & Bars \\
\hline Bezák, Mária & Chinorán & Nyitra \\
\hline Pillusz, Julianna & Szentantal & Gömör \\
\hline Kamjen, Mária & Baksa & Turóc \\
\hline Kivala, Pál & Apátfalu & Trencsén \\
\hline Kocián, Erzsébet & Szakolca & Nyitra \\
\hline Lupál, Katalin & Garamszöllös & Bars \\
\hline Molnár, Kálmán & Visk & Hont \\
\hline Ribánszky, Imre & Magyar-Soók & Nyitra \\
\hline Skarba, Ede & Bars-Rudnó & Bars \\
\hline Zeman, Mária & Szentpéter & Nyitra \\
\hline
\end{tabular}

Tabelle Nr. 4: Die Namensliste der Schülerinnen und Schüler der ersten Klasse des Gehörloseninstituts von Körmöcbánya aus den Jahren 1904-1905 (Mitteilungsblatt des Gehörloseninstituts von Körmöcbánya 1904-1905)

\begin{tabular}{|l|l|l|l|l|l|l|}
\hline Ackerbauer & Tagelöhner & $\begin{array}{l}\text { Gruben-ar- } \\
\text { beiter }\end{array}$ & $\begin{array}{l}\text { Zimmer- } \\
\text { mann }\end{array}$ & Maurer & Schuster & Insgesamt \\
\hline 4 & 4 & 1 & 1 & 1 & 1 & 12 \\
\hline
\end{tabular}

Tabelle Nr. 5: Die Beschäftigung der Eltern der Zöglinge der ersten Klasse des Gehörlosen-instituts von Körmöcbánya aus den Jahren 1904-1905 (Mitteilungsblatt des Gehörloseninstituts von Körmöcbánya, 1904-1905)

Wie es in der Zielsetzung des Instituts festgeschrieben wurde, hat man in erster Linie - mit wenigen Ausnahmen (Hont, Nógrád, Zólyom) - Schülerinnen und Schüler aus den nordwestlichen Komitaten Oberungarns bzw. aus dem Burgkomitat Bars aufgenommen.

Aus den Beschäftigungsdaten der Eltern ist ersichtlich, dass die Zöglinge dem Kleinbürgertum, der Bauernschaft und der Arbeiterschaft entstammten. Auf ihre wirtschaftliche Lage weist der Umstand hin, dass von den 9 Lernenden der ersten Klasse 6 Personen vollumfängliche Bedürftigenhilfe erhielten, 2 Personen bekamen Plätze der Stiftung des Burgkomitats Turóc bzw. 1 Person wurde auf den Platz der Stiftung des Schatzamtes aufgenommen. Ab 1906 mussten die Angestellten der staatlichen Bergbauverwaltung aufgrund der Verordnung Nr. 346/1906 des Ungarischen Sachverständigenrates für Heilpädagogik ihre gehörlosen Kinder nach Körmöcbánya schicken.

Wenn wir uns ihren schulischen Lernfortschritt anschauen, dann waren hinsichtlich des Benehmens alle 9 Schülerinnen und Schüler vorbildlich, bzw. was den Fleiß betrifft, waren fünf von innen ausdauernd, und jeweils 2 Lernende schlossen ihre schulische Laufbahn mit „dürftigem” und wechselhaftem Fleiß ab. 
Im 19. Jahrhundert wurden in der Gehörlosenbildung zwei Lehrmethoden für das Unterrichten der Gehörlosen genutzt. Die erste Methode stützte sich auf die Gebärdensprache. Das wichtigste Charakteristikum der Gebärdensprache ist, dass sie in erster Linie die Sprache der gehörlosen Menschen ist, die sie für genau solche kommunikative Zwecke benutzen, wie die hörenden Menschen die gesprochene Sprache. Für die Anhänger der gebärdensprachlichen Methode stellte das von L'Epée im Jahr 1770 gegründete Pariser Gehörloseninstitut das Muster dar, daher wird sie auch als französische Methode bezeichnet.

Die zweite Methode gründete sich auf die Aneignung der tönenden Sprache unter vollständigem Ausschluss der Gebärdensprache. Samuel Heinicke ist es zu verdanken, dass sich die Vermittlung der Aneignung der tönenden Sprache im deutschen Sprachraum einbürgerte, wobei man sich in erster Linie vor Augen hielt, dass sich die Gehörlosen mit ihrer Nutzung besser in die Mehrheitsgesellschaft integrieren können. Da in Ungarn ab 1873 gegenüber der für den Unterricht auch bereits früher verwendeten Gebärdensprache (gemischte Methode) auch die Lehre der tönenden Sprache bei den gehörlosen Kindern verfolgt wurde, spielte so die Bewertung der Aussprache eine wesentliche Rolle bei der Beurteilung des schulischen Fortschritts.

In den Mitteilungsblättern der Schulen wurden in jedem Schuljahr kurz die beim Lernen erzielten Ergebnisse der Schülerinnen und Schüler charakterisiert. Es wurde in jedem Fall festgehalten, wenn eine/ $r$ der Lernenden eine Klassenstufe wiederholen musste. In den meisten Fällen verursachte das Vorhandensein einer anderen Behinderung die ungenügenden schulischen Leistungen. „Von den Lernenden mit ungenügenden Leistungen konnten mehrere infolge einer Zurückgebliebenheit oder Schwachsinnigkeit nicht mit dem Lehrstoff der Klasse zurechtkommen" - ist im Mitteilu1ngsblatt des Instituts über das Schuljahr 1909-1910 zu lesen. (A növendékek elömenetele 1910)

Wo die Eltern landwirtschaftliche Arbeit verrichteten (Ackerbauer, Tagelöhner), dort kam es mehrfach vor, dass die Eltern selbst das Kind vorzeitig aus dem Institut herausnahmen, damit es der Familie bei der landwirtschaftlichen Arbeit helfen konnte. (Klis, 1901)

Die Lehrkräfte des Gehörloseninstituts von Körmöcbánya taten sich über die „gewöhnlichen” Gehörlosenunterrichtsmethoden hinaus auch durch mehrere Neuerungen hervor: eine davon war die Gründung einer Schülerzeitung.

„Institutszeitung [Intézeti Újság]” war der Name des im Institut von Körmöcbánya im Jahr 1912 gegründeten Wochenblattes des Jugendkreises, das in der Redaktion des Lehrers István Adamcsik von Oktober 1912 bis Mai 1914 in Körmöcbánya herausgegeben wurde. In die Zeitung kam ,in ihrem eigenen naiven Ton” jedes schulische Ereignis. Es gibt darin Spiele, die Chronik des Jugendkreises, Nachrichten, usw. Schauen wir uns ein paar Nachrichten an: „Nagy und Vancsó sind immer noch krank. - Zima sah in der vergangenen Woche ein Automobil auf dem Hauptplatz. - Der Ernährer von Mária Zsabka ist verstorben. - Die Bulgaren haben Drinápoly [Edirne, Türkei] besetzt. Lobok hat während der Osterfeiertage einen $6 \mathrm{~kg}$ schweren Kuchen aufgegessen. " - Sie vergnügen sich mit solchen Rätselfragen: Wie viel Arten Salz gibt es? Es gibt Küchensalz [konyhasó], Viehsalz 
[marhasó] und Nrisó (der Familienname eines Schülers). Was ist der Unterschied zwischen dem Wetter und Vince Uhrin? - Das Wetter ist jetzt gut, und Uhrin ist schlecht." (Tanügyi érdekesség 1913)

Mitglieder des Jugendkreises konnten jene Schülerinnen und Schüler werden, die die 4. bis 8 . Klasse besuchten, über das Schreiben der Zeitung hinaus fanden „Versammlungen, Rezitationen und Gespräche” im Jugendkreis statt. Nach Ansicht der Lehrkräfte des Gehörloseninstituts erwiesen sich die Zeitung und deren Jugendkreis als derart hervorragende Erziehungsmittel, dass die Lehrer aus anderen Gehörloseninstituten des Landes kamen, um sie zu studieren. (Ifjúsági kör 1912)

\section{Nach dem Abschluss der schulischen Laufbahn im Gehörloseninstitut}

Das zur Jahrhundertwende monatlich erscheinende Taubstummen-Blatt [Siketnémák Közlönye] veröffentlichte in jeder Ausgabe Aufrufe an die Absolventinnen und Absolventen der Gehörloseninstitute. Wenn wir uns die Aufrufe des Jahres 1901 anschauen, beziehen sie sich in erster Linie auf die auch Fingerfertigkeit erfordernden Bereiche, es war möglich, sich unter anderem als Lehrling für die Berufe Schneider, Winzer, Steinmetz und Werkzeugmacher zu bewerben. Im September 1901 veröffentlichte das Blatt einen Aufruf ,,an die gelernten und selbständigen Taubstummen", damit sie als positives Beispiel vor der Gesellschaft Ungarns ihr Namensverzeichnis und ihre Berufe veröffentlichen würden. (Tanult és önálló siketnémák névjegyzéke, 1901) Auf den Listen waren in der Mehrzahl die folgenden Berufe aufgeführt: Tischler, Schriftsetzer, Ledergraveur, Schuster, Fotograf, Konditor, Polsterer, Schneider, Sattler, Bildhauer und Dreher. (Siketnémák Közlönye, Dezember 1901; April 1902)

Diese Zeitschrift hat darüber hinaus auch mehrere Artikel mit dem Thema der Berufswahl veröffentlicht. In einem ihrer Leitartikel wurde Folgendes formuliert: „Die Institute und Schulen der Taubstummen geben ihren Zöglingen vorwiegend nur eine theoretische Ausbildung. Es kann nicht ihre Aufgabe sein, die Taubstummen zu fertigen Handwerkern oder für andere berufliche Laufbahnen zum Broterwerb heranzubilden. [...] Ihnen einen Handwerksberuf oder einen anderen Verdienstzweig zu vermitteln, ist Aufgabe der Eltern, der Vormünder und der Gesellschaft." Der Artikel stellt hiernach jene Berufe vor, die die tauben Jungen erlernen können: Schneider, Schuster, Tischler, Dreher, Korbflechter, Töpfer, Buchbinder, Schlosser, Blechner, Sattler, Gärtner, Maler und Lackierer, Schriftsetzer, Fotograf und Bildhauer. Die Darlegung wurde in Bezug auf das andere Geschlecht fortgesetzt: Sticken, Herstellung von Bett- und Unterwäsche, Nähen von Bekleidung, Maschinenstricken, Bügeln und Mangeln. Der Verfasser des Artikels hebt hervor, dass die Gehörlosen infolge ihrer herausragenden Beobachtungsfähigkeit, Genauigkeit und Gewissenhaftigkeit ,,bessere Arbeiter sind als die Menschen gesunder Empfindung.” Er zitiert auch die Ansicht eines Handwerksmeisters: „Ein Meister, der einmal einen taubstummen Lehrling hatte, wird auch ein anderes Mal gerne einen solchen als Lehrling einstellen". (Borbély 1902)

Auf der am 30. März 1908 abgehaltenen Aufsichtsratssitzung des Erfolgsinstituts von Körmöcbánya wurde zuerst geäußert, dass es erforderlich sei, zur 
Förderung der Weiterbildung und der Anstellung von Gehörlosen einen Verein zu gründen. Im Jahr 1909 wurde in Körmöcbánya der „Verein zur Unterstützung der Taubstummen" [Siketnémákat Gyámolitó Egyesület] gegründet, der zum ersten Mal mit der Genehmigung des Ministeriums eine landesweite Sammelaktion für das Institut auf den Weg brachte. Im Aufruf ist folgendes zu lesen: „Er [der Verein] stellte sich also zur Aufgabe: a) die Veranlassung der schulischen Ausbildung der im schulpflichtigen Alter befindlichen Taubstummen, b) die finanzielle und moralische Unterstützung der Absolventen des Instituts, c) die Errichtung von Beschäftigungswerkstätten für diejenigen - insbesondere Mädchen -, die keine Arbeit finden, d) Versorgung und Unterstützung derjenigen Taubstummen in Wohnheimen, die verlassen wurden und arbeitsunfähig sind. (Váradi 1912)

Dem gesetzten Ziel entsprechend hat der Verein 3 gehörlose Mädchen (Anna Bezák, Mária Csambala, Mária Zemán), die zu den besten Schülerinnen des Instituts gehörten und ihren Bildungsgang im Institut von Körmöcbánya im Schuljahr 1910-1911 absolviert hatten, teilweise in der Maschinenstrumpfstrickereiwerkstatt bzw. bei einem Schneider sowie Bett- und Unterwäschehersteller untergebracht, um dort berufliche Praxis zu erwerben, wobei der Verein ihre Unterkunft und Verpflegung sowie inr Lehrgeld bezahlte. Als der Verein die bei den Gehörloseninstituten in Kecskemét und Szeged betriebenen Nähereien begutachtete, setzte man sich zum Ziel, auch beim Institut in Körmöcbánya einen Beschäftigungsbetrieb zu errichten. (Váradi 1912)

Bei den Jungen, die die 8 Klassen des Instituts absolviert hatten, war die Situation leichter, denn die Bergbaustadt Körmöcbánya bot zahlreiche Anstellungsmöglichkeiten. Der sichereren Anstellung der Absolventen diente auch die Errichtung einer Schule für Handwerkslehrlinge. Der Ungarische Sachverständigenrat für Heilpädagogik ordnete mit seiner Verordnung Nr. 269/1907 die „Einrichtung von Berufsbildungs- und Weiterbildungslehrgängen für taubstumme Handwerkslehrlinge” an. Über ihre Realisierung können wir im Mitteilungsblatt des Gehörloseninstituts für das Schuljahr 1911-1912 Folgendes lesen: „In diesem Jahr haben wir den Lehrgang für Handwerkslehrlinge eröffnet, der einige Zeit pausierte. Von den 5 Zöglingen des Lehrgangs gelang es 4 Kinder, die das Institut im vergangenen Jahr verließen, in lokalen Handwerkswerkswerkstätten unterzubringen. Die Mädchen, die das Institut absolviert hatten, wurden vom Verein zur Unterstützung der Taubstummen gefördert. Er bezahlte für sie die Unterkunfts- und Verpflegungskosten und für eines von innen das Lehrkosten. Leider mussten wir jedoch zwei Absolventinnen nach Hause schicken. Weder die Arbeitsgelegenheiten noch die Unterkunft bieten ausreichend Sicherheit zur Verwirklichung der schönen Programme des Vereins. Dies wäre nur in seinen eigenen Werkstätten entsprechend sicherzustellen."

Im Jahr 1910 wurde die Frage geregelt, wie lange die gehörlosen Handwerkslehrlinge die Berufsschule besuchen müssen und können. Es wurde nämlich ausgesprochen, dass zwar auch die Berufsschule für Handwerkslehrlinge drei Schuljahre umfasst, kann der Lehrling im Sinne der Bestimmungen in $§ 82$ des Gesetzesartikels Nr. XVII aus dem Jahr 1884 so lange in der Berufsschule für Handwerkslehrlinge bleiben, bis seine Lehrzeit beim Handwerksmeister dauert. (Gyógypedagógiai intézmények müködése 1910) 


\section{Zusammenfassung}

Im 19. Jahrhundert boten sich für die in Oberungarn lebenden gehörlosen Kinder lange Zeit keine oder nur in einer ziemlich großen Entfernung Möglichkeiten zu lernen. In der damaligen Gesellschaft waren den Gehörlosen gegenüber zahlreiche negative Attitüden vorherrschend: Man kann nicht mit ihnen kommunizieren, daraus folgt, dass man sie nicht unterrichten kann, die Familie muss sie ernähren, sie müssen betteln.

Mit der Gründung des Instituts in Jolsva im Jahr 1901, des Instituts in Körmöcbánya im Jahr 1903, des Instituts in Pozsony im Jahr 1905 setzte sich ein Prozess in Gang, der es den Gehörlosen Oberungarns ermöglichte, eine Bildung zu erhalten, nach deren Absolvierung sie mit der Aneignung irgendeines Berufs das Fundament für ihre Zukunft setzen können und der Gesellschaft nicht ausgeliefert sind.

In den Mitteilungsblättern der Gehörloseninstitute und in der Hörgeschädigten-Presse, in den pädagogischen Fachzeitschriften jener Zeit manifestierte sich ebenfalls das Ziel, dass so viele Gehörlose wie möglich in den Unterricht gelangen und dadurch „,nützliche Mitglieder” der Gesellschaft werden sollen.

Über den Betrieb des Gehörloseninstituts von Körmöcbánya, die dort lernenden gehörlosen Kinder und ihren schulischen Werdegang kann aufgrund der Mitteilungsblätter nur bis zum Schuljahr 1911-1912 berichtet werden.

Neben den statistischen Datenreihen in den Mitteilungsblättern der Gehörloseninstitute von Körmöcbánya und den Artikeln, in denen der Bildungszweck der Schule dargestellt wird, bietet die Beschreibung, der Arbeit der im Jahre 1907 innerhalb der Mauern der Institute eingerichteten „Vereine zur Unterstützung der Taubstummen" eine Grundlage für die Erforschung der sozialen Mobilität gehörloser Schüler.

Die in der Studie aufgezeichneten Daten zeigen, dass Gehörloseinstitute für die positive soziale Mobilität gehörloser Kinder im schulpflichtigen Alter im Oberungarn eine wichtige Rolle spielten.

Die Wirksamkeit der Gehörlosenbildung wurde durch die Entwicklung eines Netzwerks für Gehörlosenbildung beschleunigt. In der gehörlosen Presse wurde eine Reihe von Missverständnissen für die Mehrheitsgesellschaft auf der Ebene der Verbreitung ausgeräumt. Danach begann allmählich eine positive Attitüde der sozialen Einstellungen.

Weitere Daten können aus Archiv-, Pressequellen gewonnen werden, deren Erschließung die geplante Fortsetzung der Arbeit sein wird.

\section{Literaturverzeichnis}

A növendékek elömenetele (1910): A Siketnémák Körmöczbányai Áll. s. Intézetének értesítője. 1909-1910. Bd. 10. Budapest: Országos Pedagógiai Könyvtár és Múzeum. E1713. A növendékek felvétele, ideje és módja (1904): A Siketnémák Körmöczbányai Áll. s. Intézetének értesítője. 1903-1904. Budapest: Országos Pedagógiai Könyvtár és Múzeum. E1713. 23-26. p. 
Az elemi népoktatás enciklopédiája (1915): Szerk.: Körösi Henrik-Szabó László. III. köt. Budapest: Franklin-Társulat.

Borbély Sándor (1902): Pályaválasztás. Siketnémák közlönye. 2/7, 153-154. p.

Borbély Vidor (1908): Siketnémák kisdedóvója. Néptanítók lapja, 308-309/11, 6-7. p.

Gyógypedagógiai intézmények müködése. (1910): A M. Kir. Kormány 1910. évi müködéséröl és az ország közállapotairól szóló jelentés és statisztikai Évkönyv, Budapest: Athenaeum Irodalmi- és Nyomda Rt.

Hercsuth Kálmán (1904): Az intézet keletkezése és az elmúlt két tanév története. $A$ Siketnémák Körmöczbányai Áll. s. Intézetének értesítője. 1903-1904. Budapest: Országos Pedagógiai Könyvtár és Múzeum. E1713. 1-8. p.

Ifjúsági kör (1912): A Siketnémák Körmöczbányai Áll. s. Intézetének értesítője. 19111912. Budapest: Országos Pedagógiai Könyvtár és Múzeum. E1713. 3-4. p.

Iparos siketnémák névjegyzéke (1902): Siketnémák közlönye. 2/4, 135-136. p.

Klis Lajos (1901): Közérdekü tudnivalók siketnémákról. Siketnémák Közlönye. 1/4, 3436. p.

Népszámlálás, 1869. https://library.hungaricana.hu/hu/view/NEDA_1870/?p$\mathrm{g}=561$ \&layout=s\&query=term\%C3\%A9szeti\%20hi\%C3\%A1 nyok Abgerufen am 01. 03 . 2020.

Népszámlálás, 1880. https://library.hungaricana.hu/hu/view/NEDA_1881_01/?p$\mathrm{g}=809$ \&layout=s\&query=testi-lelki Abgerufen am 01. 03. 2020.

Népszámlálás, 1890.

https://library.hungaricana.hu/hu/view/NEDA_1891_01/?pg=190\&layout=s\&query=siketn\%C3\%A9ma Abgerufen am 01. 03. 2020.

Népszámlálás, 1900. https://library.hungaricana.hu/hu/view/NEDA_1900_03/?p$g=739$ \&layout=s\&query=siketn\%C3\%A9ma Abgerufen am 01. 03. 2020.

Szepessyné Judik Dorottya (2009): A hallássérült emberek magyarországi mozgalmának története nemzetközi kitekintéssel. In: A fogyatékosságügy hazai és nemzetközi története. Hegedüs Lajos-Ficsorné Kurucz Margit-Szepessyné Judik Dorottya-Pajor EmeseKönczei György. ELTE Bárczi Gusztáv Gyógypedagógiai Kar. Budapest. 4-33. p.

https://mek.oszk.hu/09900/09972/09972.pdf. Abgerufen am 16. 03. 2020.

Tanult és önálló siketnémák névjegyzéke (1901): Siketnémák közlönye. 1/ 9, 80. p.

Tanügyi érdekesség, 1913: Esztergom, 137. 1913. április 27. 5.p.

Váradi Zsigmond (1912): A Siketnémákat Gyámolító Egyesület titkári jelentése. Siketnémák Körmöczbányai Áll. s. Intézetének értesítője. 1911-1912. Budapest: Országos Pedagógiai Könyvtár és Múzeum. E1713. 52-57.p. 